\title{
Clinical outcomes and differential effects of PI3K pathway mutation in obese versus non-obese patients with cervical cancer
}

\author{
Perry Grigsby ${ }^{1,2,3}$, Adnan Elhammali ${ }^{4}$, Fiona Ruiz ${ }^{1}$, Stephanie Markovina ${ }^{1,2}$, Michael \\ D. McLellan ${ }^{5}$, Christopher A. Miller ${ }^{5}$, Anupama Chundury ${ }^{1}$, Ngoc-Anh L. Ta ${ }^{6}$, \\ Ramachandran Rashmi ${ }^{1}$, John D. Pfeifer ${ }^{7,8}$, Robert S. Fulton ${ }^{5}$, Todd DeWees ${ }^{1}$ and \\ Julie K. Schwarz ${ }^{1,2,9}$ \\ ${ }^{1}$ Department of Radiation Oncology, Washington University School of Medicine, St. Louis, MO, USA \\ ${ }^{2}$ Alvin J. Siteman Cancer Center, Washington University School of Medicine, St. Louis, MO, USA \\ ${ }^{3}$ Division of Nuclear Medicine, Mallinckrodt Institute, Washington University School of Medicine, St. Louis, MO, USA \\ ${ }^{4}$ Department of Radiation Oncology, MD Anderson Cancer Center, University of Texas Health Science Center, Houston, TX, USA \\ ${ }^{5}$ McDonnell Genome Institute, Washington University School of Medicine, St. Louis, MO, USA \\ ${ }^{6}$ Saint Louis University School of Medicine, St. Louis, MO, USA \\ ${ }^{7}$ Department of Pathology and Immunology, Washington University School of Medicine, St. Louis, MO, USA \\ ${ }^{8}$ Department of Obstetrics and Gynecology, Washington University School of Medicine, St. Louis, MO, USA \\ ${ }^{9}$ Department of Cell Biology and Physiology, Washington University School of Medicine, St. Louis, MO, USA \\ Correspondence to: Julie K. Schwarz, email: jschwarz@wustl.edu
}

Keywords: obesity; cervical cancer; PI3K; PTEN; mutation

Received: September 07,2017 Accepted: December 13,2017 Published: December 23, 2017

Copyright: Grigsby et al. This is an open-access article distributed under the terms of the Creative Commons Attribution License 3.0 (CC BY 3.0), which permits unrestricted use, distribution, and reproduction in any medium, provided the original author and source are credited.

\section{ABSTRACT}

The purpose of this study was to evaluate the effect of obesity and obesityassociated factors on the outcomes of patients with cervical cancer. Outcomes were evaluated in 591 patients with FIGO Ib to IV cervical cancer treated uniformly with definitive radiation. Patients were stratified into 3 groups based upon pretreatment Body Mass Index (BMI): A $\leq 18.5$; $B$ 18.6 - 34.9; and $C \geq 35$. The 5-year freedom from failure rates were 58,59 , and $73 \%$ for $B M I$ groups $A, B$, and $C(p=0.01)$. Overall survival rates were 50,59 , and $68 \%$, respectively $(p=0.02)$. High expression of phosphorylated AKT (PAKT) was associated with poor outcomes only in non-obese patients. Obese patients with PI3K pathway mutant tumors had a trend toward favorable outcomes, while a similar effect was not observed in non-obese patients. Compared to similar tumors from non-obese hosts, PIK3CA and PTEN mutant tumors from obese patients failed to express high levels of phosphorylated AKT and its downstream targets. These results show that patients with obesity at the time of diagnosis of cervical cancer exhibit improved outcomes after radiation. PI3K/AKT pathway mutations are common in obese patients, but are not associated with activation of AKT signaling.

\section{INTRODUCTION}

Increasing body mass index (BMI) has been associated with increased cancer-related mortality. Calle and colleagues found that overweight women had an increased risk of death from all causes and that the death rate in women from cervical cancer increased with increasing BMI [1]. In the UK's Million Women cohort study, Reeves and associates found that cervical cancer incidence and mortality increased with increasing 
BMI [2]. However, $\mathrm{Xu}$ and colleagues reported results from the Cancer Genome Atlas (TCGA) showing lower mortality in cervical cancer patients with higher BMI values [3]. Although the analysis by Xu using TCGA data demonstrated lower mortality in cervical cancer patients with higher BMI values, these patients were treated with a variety of techniques and outcome data was not prospectively collected. The aim of the current study was to use a well-annotated clinical database to evaluate the association of obesity and cervix cancer patient outcomes after uniform treatment with curative intent radiation. A prospectively collected institutional tumor bank was used to determine whether obesity associated factors (metformin, insulin, and PI3K/AKT signaling) were associated with outcomes in the context of definitive radiation treatment.

\section{RESULTS}

Patient baseline characteristics are shown in Table 1. There were no significant differences in the traditional patient- and tumor-related prognostic factors of age, tumor stage, pretreatment ${ }^{18} \mathrm{~F}$-fluoro-deoxy-glucose (FDG) positron emission tomography (PET) lymph node (LN) status and histology relative to the patient's BMI. Patients with a high BMI were more likely to have Type II diabetes, take metformin, and take insulin. The distribution of patient BMI is shown in Figure 1. We have previously reported that cervical tumor FDG uptake quantified by Standardized Uptake Value (SUV) on pretreatment FDGpositron emission tomography (PET) scans is associated with poor prognosis [4]. To determine whether obesity remained a significant predictive factor in the setting of other known pretreatment risk factors, including cervical tumor SUV, a multivariate analysis was performed (Table 2). Cox proportional hazards modeling demonstrated that obesity remained significant in a model that included clinical stage, cervix tumor SUV and pretreatment lymph node status. There was no association between patient obesity and individual pretreatment risk factors including clinical stage, lymph node status and cervix tumor SUV. Similarly, there was no association between tumor human papilloma virus (HPV) status and patient obesity (data not shown).

Overall survival outcomes are shown in Figure 2A. Overall survivals at 5 years were significantly improved for obese patients treated with radiation $(50,59$, and $68 \%$ for BMI groups $\mathrm{A}, \mathrm{B}$, and $\mathrm{C} \mathrm{p}=0.02)$. Corresponding 5 -year FF rates were 58, 59, and 73\% respectively (Figure $2 \mathrm{~B}, \mathrm{p}=0.01)$. Metformin use was more common in BMI Group C (15\%) than in Groups A $(6 \%)$ or B $(3 \%)$; however $\mathrm{FF}$ and $\mathrm{OS}$ were not affected by metformin use (Figure $2 \mathrm{C}$ and 2D). Similarly, FF and OS were not affected by diabetes or insulin use (Figures 2E-2H).

We have previously reported that increased expression of phosphorylated AKT is associated with poor prognosis after definitive radiation for cervical cancer [5]. To determine whether activation of AKT signaling was associated with outcome in obese versus non-obese patients, we analyzed expression of phosphorylated AKT (pAKT) by immunohistochemistry (IHC) using a commercially available antibody specific for AKT phosphorylated at serine 473 (S473). High pAKT expression by IHC was not associated with a statistically significant decrease in FF amongst all-comers $(\mathrm{p}=0.17$, Figure 3A). High pAKT expression was associated with significantly reduced FF only in patients with $\mathrm{BMI} \leq 30$ $(\mathrm{p}=0.05$, Figure $3 \mathrm{~B})$. In contrast, in the cohort of patients

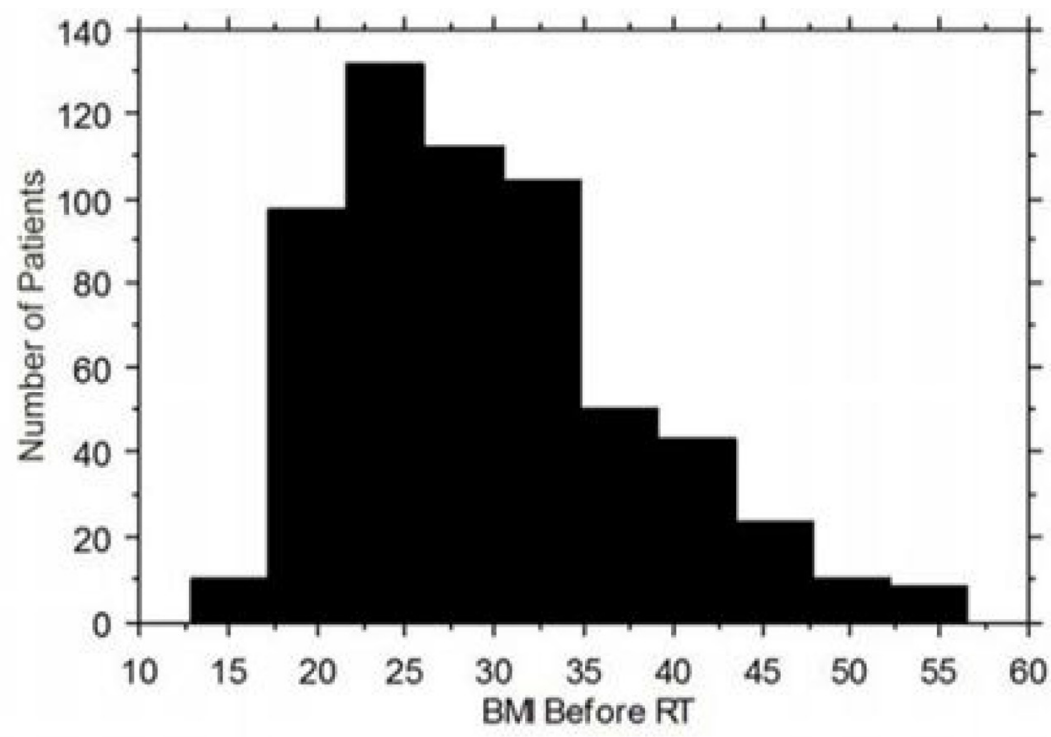

Figure 1: Patient characteristics and BMI distribution of the study population. Pretreatment Body Mass Index (BMI) distribution histogram for 591 patients treated with radiation for cervical cancer. 
Table 1: Patient baseline characteristics

\begin{tabular}{|c|c|c|c|c|c|}
\hline & $\begin{array}{c}\text { All } \\
\mathrm{N}=591\end{array}$ & $\begin{array}{l}\text { BMI A } \\
\leq 18.5 \\
N=33\end{array}$ & $\begin{array}{c}\text { BMI B } \\
\text { 18.6-34.9 } \\
\text { N }=427\end{array}$ & $\begin{array}{c}\text { BMI C } \\
\geq 35 \\
N=131\end{array}$ & p-value \\
\hline \multicolumn{6}{|l|}{ Age } \\
\hline Range & $23-92$ & $32-81$ & $23-92$ & $24-83$ & N.S. \\
\hline Mean & 52 & 52 & 52 & 52 & \\
\hline \multicolumn{6}{|l|}{ Stage } \\
\hline I (Ia1, Ia2, Ib1, Ib2) & 200 & 9 & 131 & 60 & N.S. \\
\hline II (IIa, IIb) & 237 & 13 & 175 & 49 & \\
\hline III (IIIa, IIIb) & 139 & 9 & 110 & 20 & \\
\hline IV (IVa, IVb) & 15 & 2 & 11 & 2 & \\
\hline \multicolumn{6}{|l|}{ BMI } \\
\hline Range & $13-57$ & $13-18.5$ & $18.6-34.9$ & $35-57$ & N.A. \\
\hline Median & 27.5 & 18 & 26.1 & 40.5 & \\
\hline \multicolumn{6}{|l|}{ PET lymph nodes (LN) } \\
\hline None & 261 & 11 & 182 & 68 & N.S. \\
\hline Pelvis & 240 & 18 & 178 & 44 & \\
\hline Pelvis \& Para-artic & 84 & 3 & 62 & 19 & \\
\hline $\mathrm{P}+\mathrm{P}+\mathrm{SCV}$ & 6 & 1 & 5 & 0 & \\
\hline \multicolumn{6}{|l|}{ Histology } \\
\hline Squamous & 502 & 29 & 368 & 105 & N.S. \\
\hline Adenocarcinoma & 60 & 2 & 37 & 21 & \\
\hline Other & 29 & 2 & 22 & 5 & \\
\hline \multicolumn{6}{|l|}{ Metformin } \\
\hline Yes & 35 & 2 & 14 & 19 & $<0.0001$ \\
\hline No & 544 & 31 & 405 & 108 & \\
\hline Unknown & 12 & 0 & 8 & 4 & \\
\hline \multicolumn{6}{|l|}{ Insulin } \\
\hline Yes & 30 & 1 & 12 & 17 & $<0.0001$ \\
\hline No & 549 & 32 & 407 & 110 & \\
\hline Unknown & 12 & 0 & 8 & 4 & \\
\hline \multicolumn{6}{|l|}{ Type II } \\
\hline Yes & 75 & 2 & 33 & 40 & $<0.0001$ \\
\hline No & 504 & 31 & 386 & 87 & \\
\hline Unknown & 12 & 0 & 8 & 4 & \\
\hline
\end{tabular}

with $\mathrm{BMI}>30$, high $\mathrm{pAKT}$ expression was not associated with FF ( $p=0.76$, Figure $3 \mathrm{C}$ ). These results imply that the significance of pAKT expression as a biomarker for poor outcome after radiation treatment depends on patient obesity. For patients with a low BMI, expression of pAKT is predictive of poor outcome after irradiation. For obese patients, pAKT expression alone is not predictive, suggesting that downstream signals from AKT have less 


\begin{tabular}{lcc}
\hline & Multivariate hazard ratio (95\% CI) & P-value \\
\hline Lymph Node Status (LN+ vs LN-) & $1.692(1.338,2.320)$ & 0.0010 \\
Cervix Standardized Uptake Value (SUV) & $1.020(1.002,1.037)$ & 0.0280 \\
Stage I (ref) & & 0.0018 \\
Stage II & $1.436(0.982,2.100)$ & \\
Stage III & $2.117(1.413,3.171)$ & 0.0388 \\
Stage IV & $2.630(1.039,6.658)$ \\
BMI $>=35$ vs BMI $<35$ & $0.655(0.439,0.979)$ & \\
\hline
\end{tabular}

impact on radiation responses when the patient is obese and the resulting tumor environment is influenced by the obese state.

Somatic mutations in the PI3K/AKT signaling pathway have been identified in many cancers and are thought to promote inappropriate activation of $\mathrm{AKT}$ signaling. To identify somatic mutations in $P I K 3 C A$ and PTEN (including PTEN copy number (CN) loss) in our patient population, targeted exome sequencing was performed on 91 pretreatment cervical tumor biopsies. The most common PIK3CA mutation was E545K in both groups (Table 3 and Figure 4A). PIK3CA and PTEN mutations were identified in 30 samples, 14 from nonobese patients and 16 from obese patients. Previous work from our group identified alterations in expression of genes from the PI3K/AKT pathway that were associated with incomplete metabolic response after chemoradiation in cervical cancer, and preliminary data demonstrated an association between PIK3CA activating mutations and inferior recurrence-free survival outcome after radiation $[5,6]$. For this reason we compared FF outcomes for obese and non-obese patients with and without PI3K pathway mutations. There was a trend toward improved FF for obese patients with PI3K pathway mutations $(\mathrm{p}=.09)$ (Figure 4B). In contrast, non-obese patients with PI3K pathway mutations experienced similar if not inferior FF outcomes compared to non-obese patients without PI3K pathway mutations $(\mathrm{p}=.23)$ (Figure 4C).

We then used reverse phase protein phosphorylation data from the Cancer Genome Atlas Project to test whether phosphorylation of AKT and AKT downstream targets was different in cervical tumors from patients who are obese versus non-obese. Among TCGA cervical cancer patients, obesity showed a trend toward improved overall survival, however this result did not reach statistical significance ( $\mathrm{p}$ $=.07$, Figure 5). We then specifically examined whether protein phosphorylation patterns for AKT and downstream targets of AKT were different in PIK3CA and PTEN mutant tumors from obese versus non-obese hosts. In nonobese hosts, PIK3CA and PTEN mutant tumors displayed statistically significant increased phosphorylation of AKT at 2 key sites critical for full activation of AKT kinase activity, S473 and threonine 308 (T308) (Figure 6). In contrast, no increase in AKT phosphorylation at either site was observed for PIK3CA and PTEN mutant tumors from patients with $\mathrm{BMI}>35$. In addition, phosphorylation of PRAS40 and TUBERIN, two downstream direct targets of AKT kinase, was increased in PIK3CA and PTEN mutant tumors from non-obese hosts $(\mathrm{p}=.03$ and $\mathrm{p}=.02$, respectively), but phosphorylation of PRAS40 and TUBERIN was not increased in PIK3CA and PTEN mutant tumors from patients with BMI $>35$. It should be noted, however, that not all AKT targets demonstrated differences in phosphorylation based upon obesity of the host (Figure 6 GSK3 and data not shown).

\section{DISCUSSION}

In this study, we report that severely obese patients with cervical cancer treated with radiation have a better survival outcome than obese, normal, and underweight patients. Most investigators report that obese patients are at a higher risk for developing certain types of cancer and that their survival outcomes are poor when compared to normal weight patients. The mechanisms for poor outcomes in obese patients include insulin resistance, hyperinsulinemia, inflammatory responses, and increased bio-availability of steroid hormones. Our clinical data for cervical cancer are unique in that obese patients, specifically severely obese patients $($ BMI > 35) treated with definitive radiation, have favorable survival outcomes after treatment. Our study represents the largest reported series to date of clinical outcomes related to obesity for cervical cancer patients.

Metformin use has been reported to both decrease cancer risk and decrease cancer mortality. In our study, the severely obese women with cervical cancer, outcomes were not affected by metformin use, type II diabetes, or insulin use. Thus, the favorable outcome results in severely obese patients cannot be explained by diabetes or metformin use. Therefore, we explored other potential biological explanations for the difference in survival outcomes related to obesity.

Previous work from our group identified alterations in expression of genes from the PI3K/AKT pathway that were associated with incomplete metabolic 
response after chemoradiation in cervical cancer, and preliminary data demonstrated an association between $P I K 3 C A$ activating mutations and inferior disease-free

A

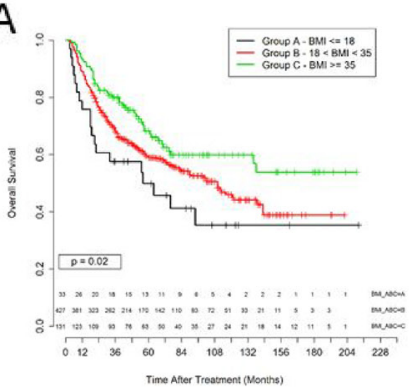

C

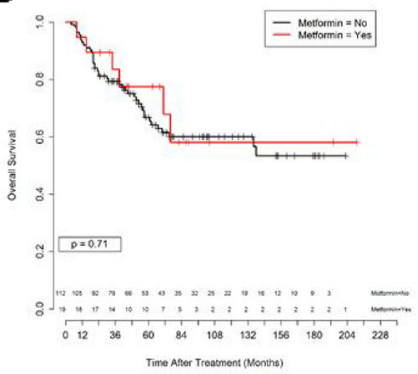

$\mathrm{E}$

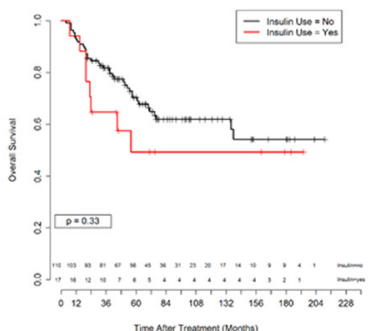

G

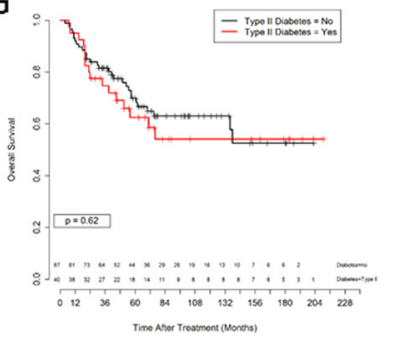

survival outcome after radiation $[5,6]$. Examination of the specific mutations present in obese and non-obese patients (Table 3 ) demonstrated roughly equal numbers
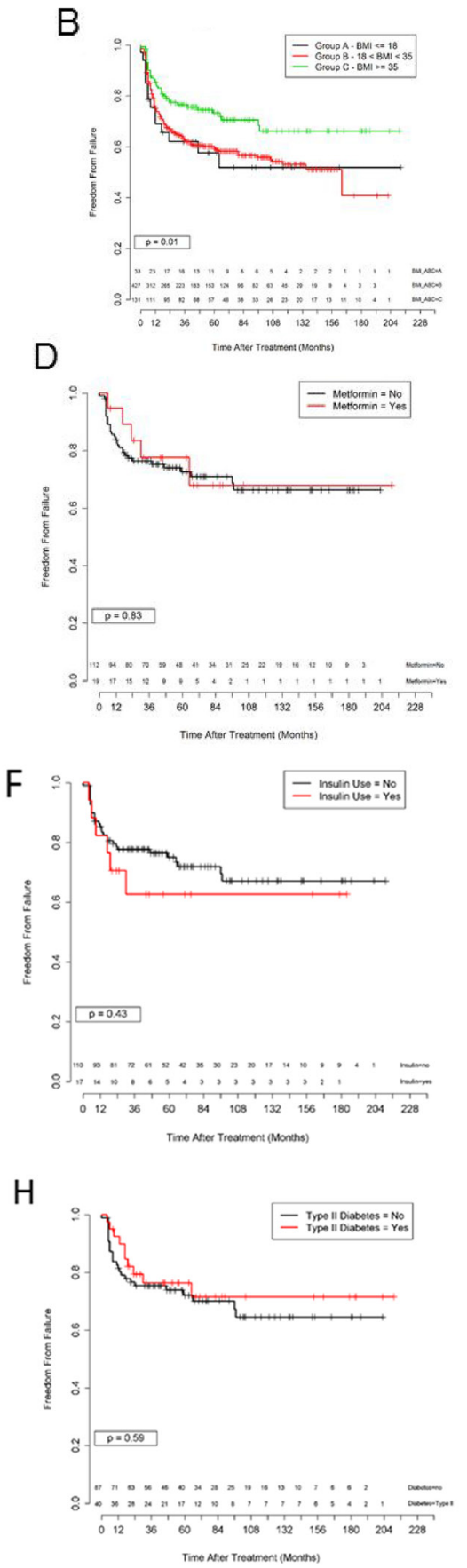

Figure 2: Survival outcomes separated by obesity and metformin use. (A) Kaplan Meier curve for overall survival for patients in BMI group: $\mathrm{A} \leq 18.5$ (black); B $18.6-34.9$ (red); and C $\geq 35$ (green), $\mathrm{p}=0.02$. C. (B) Kaplan Meier curve for freedom from failure for patients in BMI group: $\mathrm{A} \leq 18.5$ (black); B 18.6 - 34.9 (red); and $\mathrm{C} \geq 35$ (green), $\mathrm{p}=0.01$. (C) Kaplan Meier curve for overall survival for patients from BMI group $\mathrm{C}$ with (red) and without (black) metformin use ( $\mathrm{p}=0.71$ ) (D) Kaplan Meier curve for freedom from failure for patients from BMI group $\mathrm{C}$ with (red) and without (black) metformin use ( $\mathrm{p}=0.83$ ). (E) Kaplan Meier curve for overall survival for patients from BMI group $\mathrm{C}$ with (red) and without (black) insulin use ( $\mathrm{p}=0.33$ ). (F) Kaplan Meier curve for freedom from failure for patients from BMI group $\mathrm{C}$ with (red) and without (black) insulin use ( $\mathrm{p}=0.43$ ). (G) Kaplan Meier curve for overall survival for patients from BMI group $\mathrm{C}$ with (red) and without (black) a diagnosis of diabetes $(\mathrm{p}=0.62)$. $(\mathbf{H})$ Kaplan Meier curve for freedom from failure for patients from BMI group $\mathrm{C}$ with (red) and without (black) a diagnosis of diabetes $(\mathrm{p}=0.59)$. 

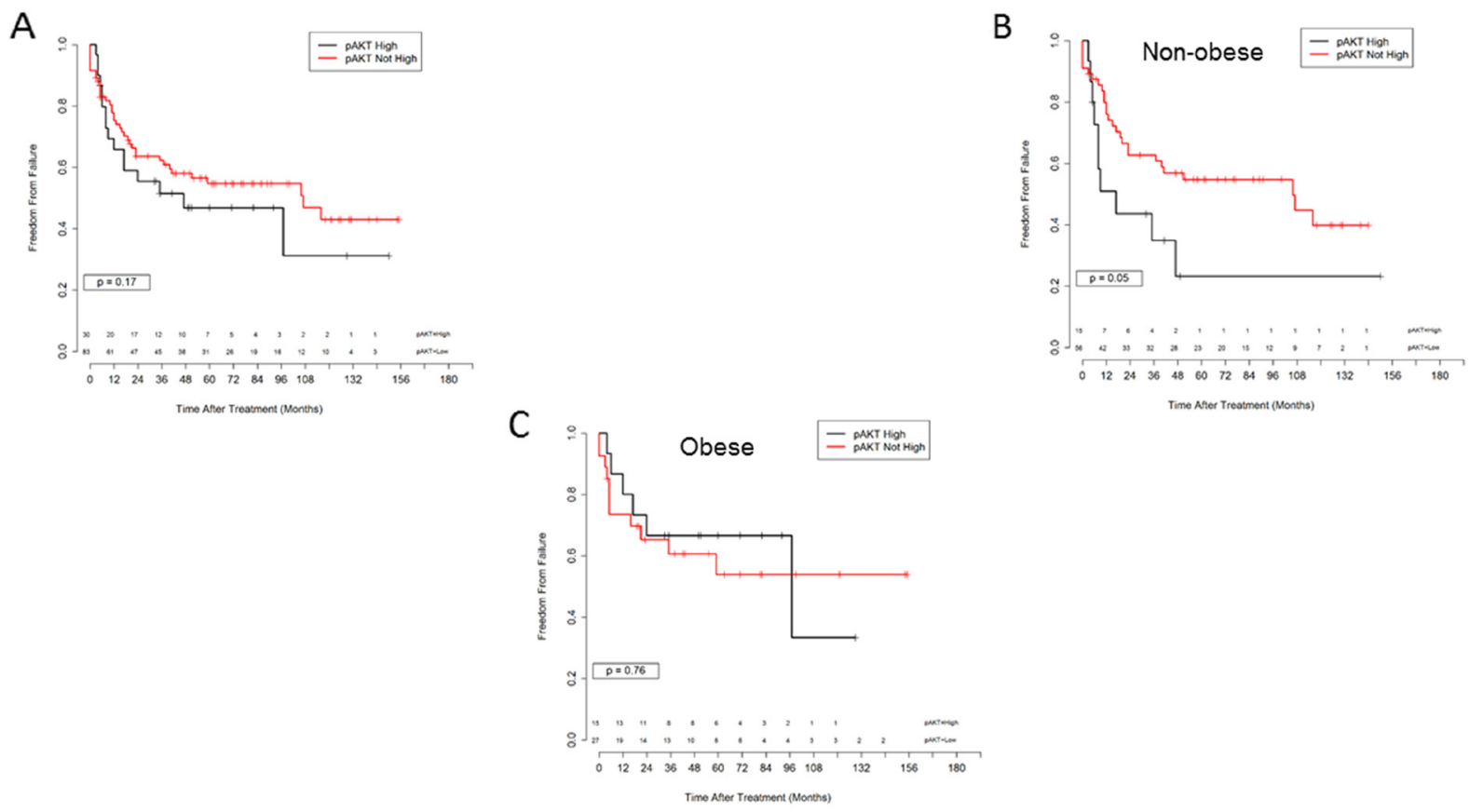

Time Ater Trestrent (Months)

Figure 3: Immunohistochemistry for pAKT and outcome in obese versus non obese patients. (A) Freedom from failure for all patients, high versus not high pAKT IHC, $p=0.17$. (B) Freedom from failure, BMI $\leq 30$, high versus not high $p A K T$ IHC, $p=0.05$. (C) Freedom from Failure, BMI $>30$, high versus not high pAKT IHC, $\mathrm{p}=0.76$.

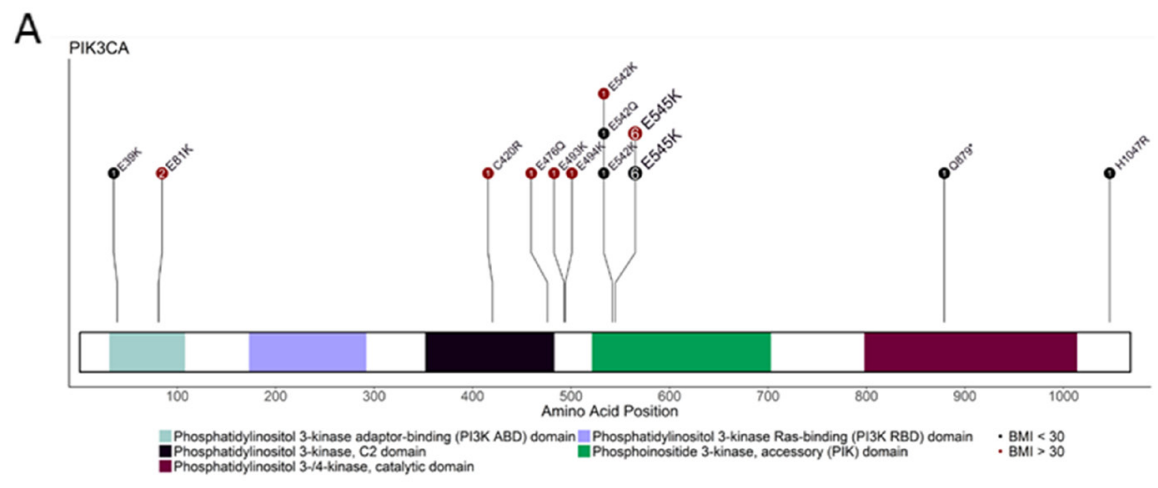

B

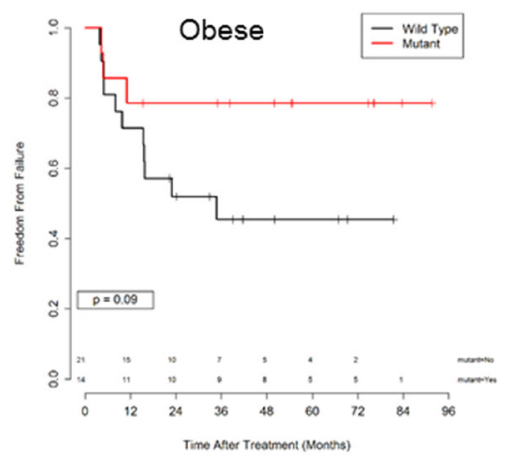

$\mathrm{C}$

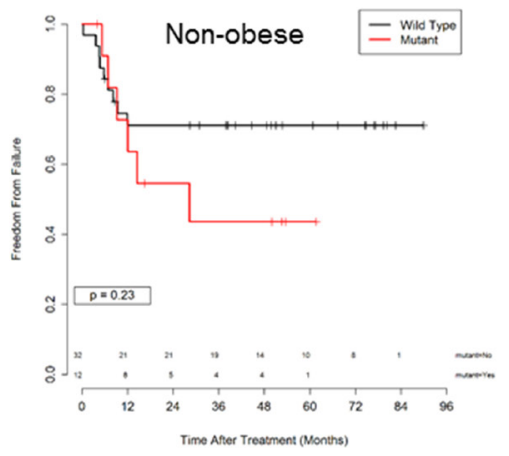

Figure 4: PI3K pathway mutations and outcome in obese versus non obese patients. (A) Distribution of PIK3CA somatic mutations in obese and non-obese patients. (B) Kaplan Meier curves for freedom from failure in obese patients (BMI $>30)$ with (red) and without (black) PIK3CA and PTEN mutations, $\mathrm{p}=.09$. (C) Kaplan Meier curves for freedom from failure in non-obese patients (BMI $<30)$ with (red) and without (black) PIK3CA and PTEN mutations, $\mathrm{p}=0.23$. 
Table 3: PIK3CA, PTEN and BMI in the WUSTL sequencing cohort

\begin{tabular}{|c|c|c|c|c|}
\hline Sample & PIK3CA & PTEN & BMI & HPV subtype \\
\hline 705973 & p.E545K & CN Loss & 18.3 & HPV 52 \\
\hline 760832 & p.E542K & WT & 18.8 & HPV 16 \\
\hline 724788 & p.E39K & WT & 23.2 & HPV 16, HPV 18 \\
\hline 727879 & p.E545K & WT & 23.2 & HPV 16 \\
\hline 740838 & p.E545K & WT & 23.2 & HPV 18 \\
\hline 704786 & p.E542Q & WT & 23.7 & HPV 16 \\
\hline 707755 & WT & CN Loss & 24 & HPV 16 \\
\hline 734998 & p.E545k, p.Q879* & WT & 24.4 & HPV 31 \\
\hline 733024 & p.H1047R & WT & 25.2 & HPV 16 \\
\hline 703822 & p.E545K & WT & 25.5 & HPV 16 \\
\hline 722396 & p.E545K & WT & 25.5 & HPV 16 \\
\hline 710808 & WT & CN Loss & 26 & Many \\
\hline 704849 & WT & CN Loss & 26.1 & HPV 16 \\
\hline 711380 & WT & CN Loss & 26.6 & Negative \\
\hline 736133 & p.E545K & WT & 31.6 & HPV 16 \\
\hline 720170 & WT & p.R233* & 31.8 & Negative \\
\hline 704821 & p.E81K & WT & 32.1 & HPV 16 \\
\hline 704956 & p.E493K, p.E476Q, p.E494K & WT & 35 & HPV 58 \\
\hline 728723 & p.E81K & WT & 35.7 & HPV 16 \\
\hline 720059 & p.E545K & WT & 37.3 & Negative \\
\hline 714694 & p.E545.K & WT & 37.6 & HPV 16 \\
\hline 731976 & WT & p.R130Q & 39.1 & HPV 16 \\
\hline 733384 & WT & CN Loss & 39.7 & HPV 33 \\
\hline 714960 & p.E542K & WT & 39.9 & HPV 16 \\
\hline 736462 & p.E545K & WT & 43.2 & HPV 16 \\
\hline 711012 & p.E545K & WT & 43.5 & HPV 16 \\
\hline 738147 & p.C420R & p.R130G, CN Loss & 45 & Negative \\
\hline 707913 & WT & CN Loss & 47.1 & HPV 16 \\
\hline 755439 & p.E545K & WT & 47.1 & Many \\
\hline 746486 & WT & p.T319fs & 56.5 & Negative \\
\hline
\end{tabular}

of PIK3CA activating and PTEN inactivating mutations between the two groups. The most common PIK3CA mutation was E545K in both groups. E545K is a common cancer-associated mutation in the helical domain of the p110alpha subunit of PI3K, which results in constitutive PI3K activity and has been reported to transform cells and enhance tumorigenic phenotypes [7-9]. A number of less well characterized mutations in PIK3CA were identified in our data set, including $\mathrm{C} 420 \mathrm{R}$, which has been reported to induce oncogenic transformation by promoting membrane binding of p110alpha [10]. The most frequent mechanism of PTEN mutation was PTEN copy number deletion. PTEN R130Q/G point mutations and a single frameshift at T319 were identified in the obese patient group.

Although obese patients had high rates of $P I K 3 C A$ and PTEN mutations, obesity was not associated with increased expression of pAKT, raising the possibility that PIK3CA and PTEN mutations in obese patients 
do not activate downstream signaling via AKT to the same extent as similar mutations in non-obese patients. Increased expression of pAKT was associated with inferior outcome after chemo-radiation only in the nonobese group, suggesting that AKT downstream signals are differentially regulated between the two groups. To further evaluate potential molecular mechanisms for the observed difference in outcome for PI3K mutant tumors in obese versus non-obese patients, we compared publically available reverse phase protein array (RPPA) data from The Cancer Genome Atlas Project (TCGA). In nonobese hosts, PIK3CA and PTEN mutant tumors displayed significantly higher levels of phosphorylation of AKT at S473 and T308, key sites critical for full activation of AKT kinase activity. In contrast, no increase in AKT phosphorylation at either site was observed for $P I K 3 C A$ and PTEN mutant tumors from patients with $\mathrm{BMI}>35$. Phosphorylation of downstream AKT targets PRAS40 and TUBERIN was increased in PIK3CA and PTEN mutant tumors from non-obese hosts, but phosphorylation was not increased in PIK3CA and PTEN mutant tumors from patients with $\mathrm{BMI}>35$. These results suggest that PIK3CA and PTEN mutations more effectively activate AKT kinase activity and phosphorylation of select AKT downstream targets in cervical tumors when the patient is non-obese.

It is important to note that in the TCGA project for cervical cancer, the majority of patients were treated with surgery for early stage disease. When we compared survival outcome data from the TCGA based on pretreatment BMI groups, we noted a similar trend of improved survival outcome with obesity, however outcomes in the TCGA cohort did not reach statistical significance $(\mathrm{p}=.07$, Figure 5$)$. There are several potential explanations for the lack of statistical significance in the TCGA cohort including smaller cohort size, shorter median follow-up time and non-uniform treatment. The effects of obesity on outcome may be different for patients

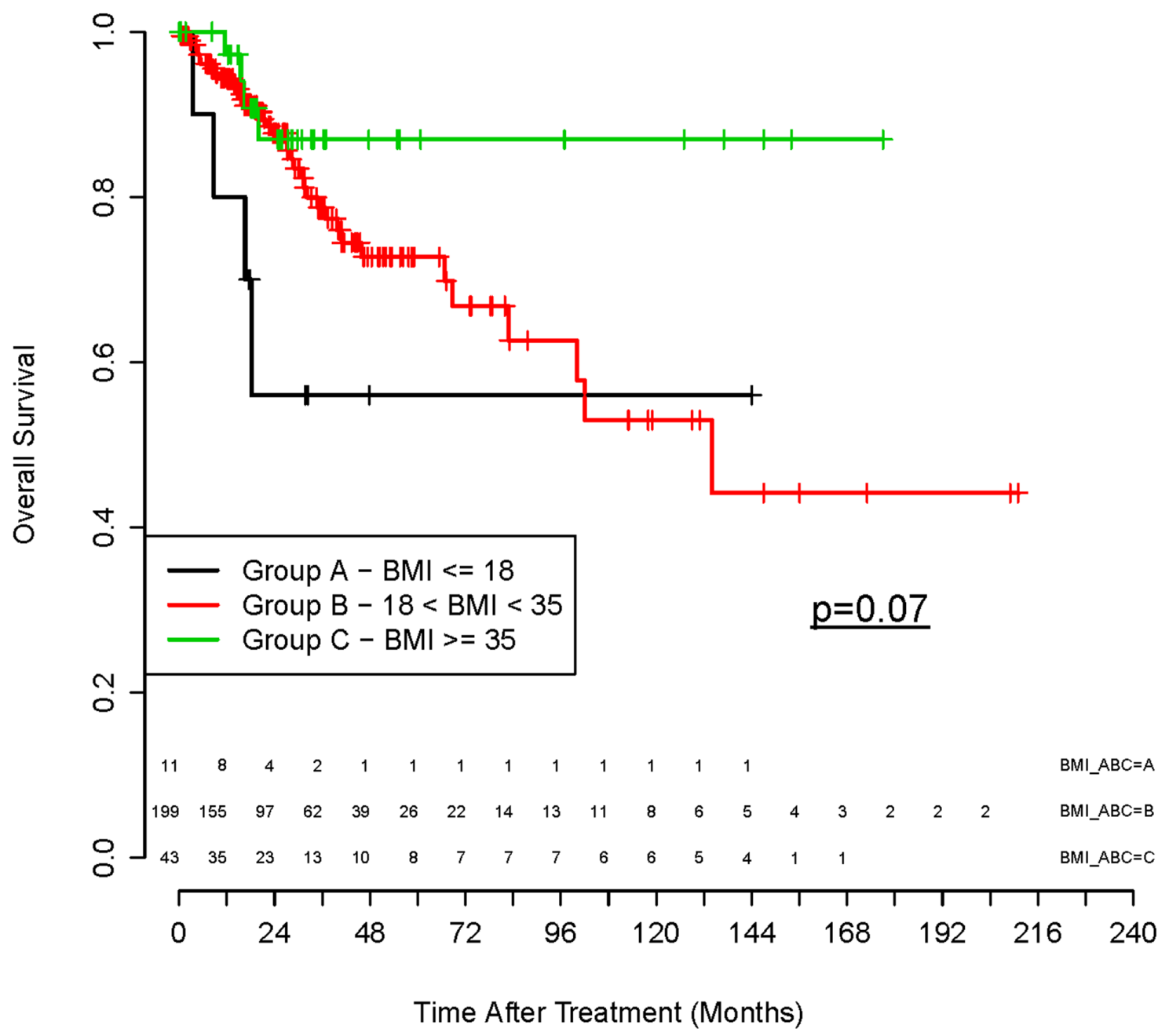

Figure 5: Overall survival outcomes from The Cancer Genome Atlas (TCGA) dataset separated by pretreatment BMI. Overall survival outcomes obtained from the TCGA dataset based on pretreatment BMI: BMI group: A $\leq 18$ (black); B 18 - 35 (red); and $\mathrm{C} \geq 35$ (green), $\mathrm{p}=0.07$. 
who are managed by primary surgery versus radiation. For example, post-surgical complications may be increased in obese patients and this may obscure any protective effects on survival outcome. Alternatively, the protective effects of obesity on clinical outcomes may be more pronounced when patients are treated with primary radiation therapy.

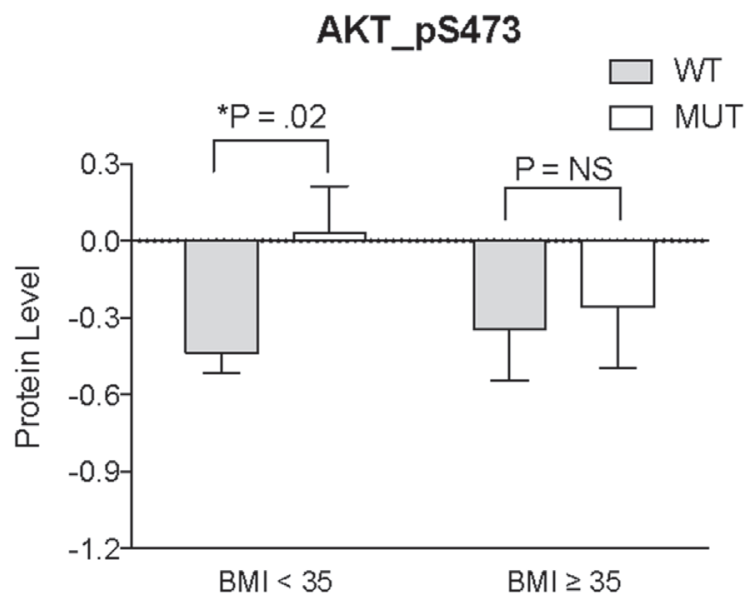

GSK3_pS9

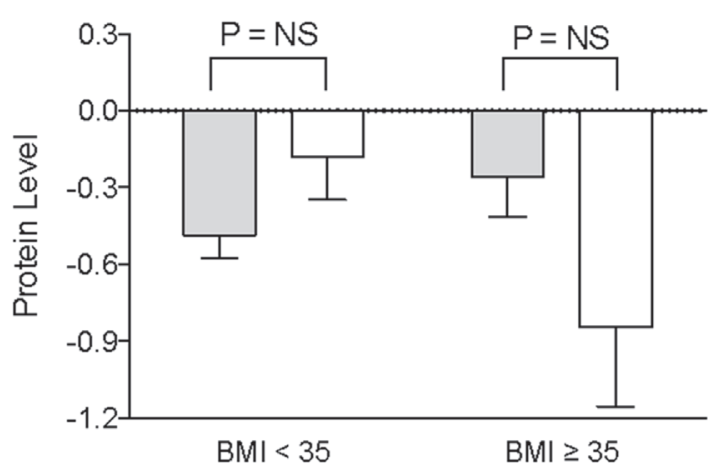

TUBERIN_pT1462

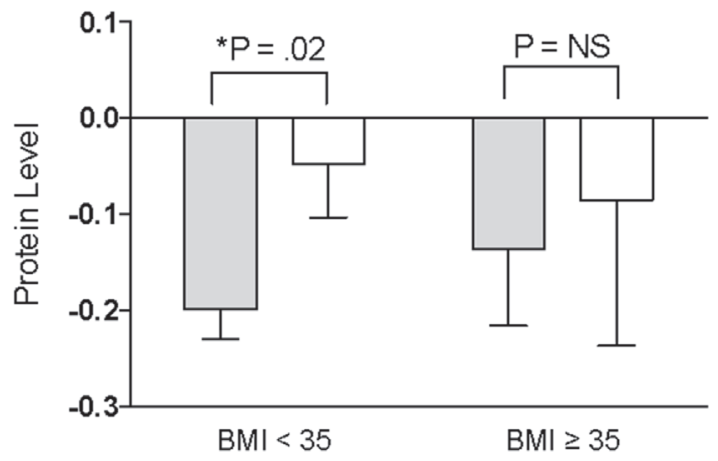

Additional study with prospectively collected clinical outcome databases will be needed to address this question.

While activation of the PI3K/AKT pathway is common in many cancers, and preclinical evidence for an oncogenic role for $P I K 3 C A$ mutations is well accepted, mutations in $P I K 3 C A$ have performed poorly as
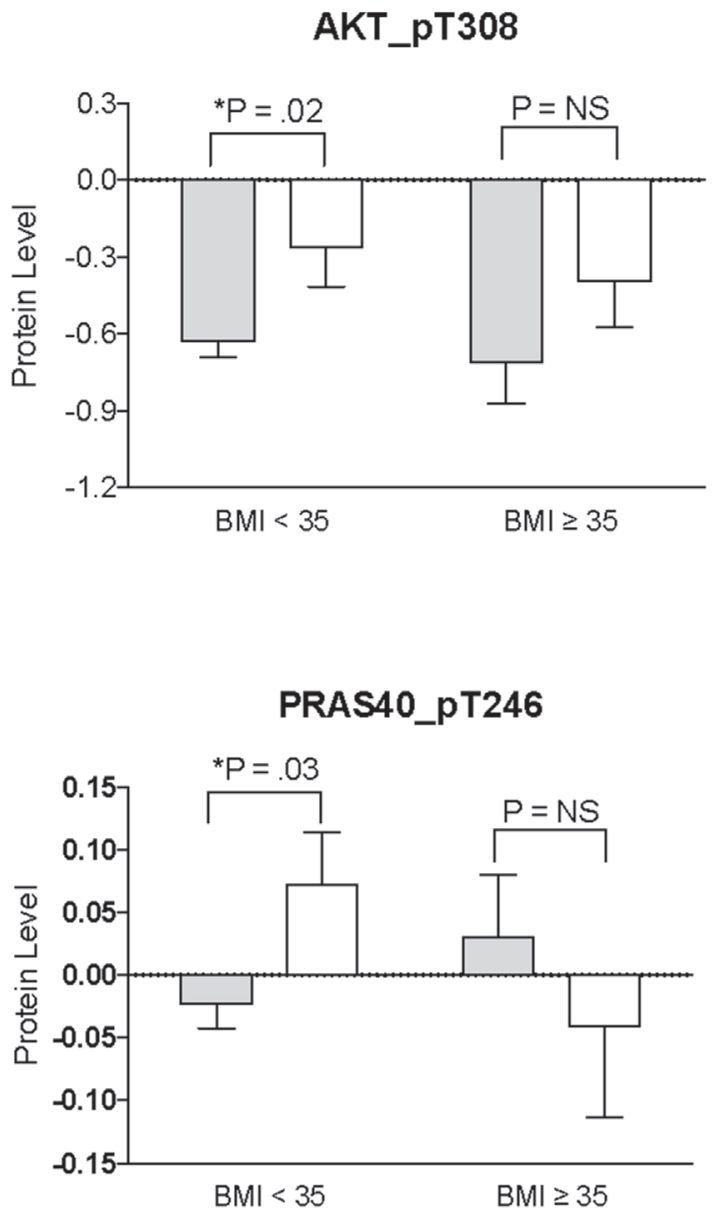

Figure 6: Phosphoprotein expression from PIK3CA and PTEN mutant tumors in obese versus non-obese hosts. Log2 normalized phosphoprotein levels were compared for AKT_pS473, AKT_PT308, PRAS40_pT246, GSK_PS9 and Tuberin_pT1462 using a two tailed $t$-test with multiple comparison correction. 
biomarkers for outcome in solid tumors [11]. Our study suggests that obesity may be one factor that influences AKT signaling downstream of an activating $P I K 3 C A$ mutation. PIK3CA and PTEN genomic mutations may be more accurate biomarkers for cervical cancer outcome only for patients who are non-obese. These results could be used to inform future clinical trial design. Stratification on the basis of PI3K pathway mutation may be more effective if the host environment is considered.

The implications of obesity and its effects on signaling at the molecular level are complex. How an obese state influences cervical tumorigenesis and the response to anti-cancer treatments, including irradiation, are incompletely understood. Our data suggest that cervical tumor cell signaling through the PI3K/AKT pathway is distinct in obese versus non-obese patients and that these differences are associated with the response to concurrent standard of care chemoradiation. Additional preclinical study is needed to understand how the obese state may influence oncogenic signaling and radiation responses.

\section{MATERIALS AND METHODS}

\section{Patient databases}

1) Clinical outcomes database $(\mathbf{N}=591)$ Patients in the study cohort consisted of 591 patients with a new diagnosis of advanced cervical cancer seen at our institution from June 1997 to June 2014. Pre-treatment BMI (calculated using the National Institute of Health online calculator) was recorded for all patients. All patients underwent a pre-treatment workup including history and physical, examination under anesthesia, and a whole-body FDG-PET/CT.

2) Immunohistochemistry cohort $(\mathrm{N}=113)$ Archived formalin-fixed, paraffin-embedded (FFPE) specimens were used to construct a tissue microarray. Prospective data collection, retrospective data collection, and use of FFPE specimens were performed with University IRB approval with waiver of informed consent (201603148, 201108070, 201201099, 201208101, 201104085, and 201601055).

3) Sequencing cohort ( $\mathbf{N}=91)$ A subset of 91 patients were prospectively enrolled into a tumor banking study at the time of initial diagnosis. This study was approved by the Institutional Review Board and all patients provided informed consent for sequencing (201105374). Tumor biopsies and blood were obtained prior to treatment and stored at the Tissue Procurement Facility.

\section{Radiation treatment}

Definitive radiation with curative intent was administered in all $(\mathrm{N}=591)$ patients. Radiation treatment consisted of both external beam radiotherapy and intracavitary brachytherapy using techniques previously described [12]. The median prescribed external irradiation dose to the pelvic lymph nodes was $50.4 \mathrm{~Gy}$. Concurrent chemotherapy (once weekly $40 \mathrm{mg} / \mathrm{m}^{2}$ cisplatin) was administered in $88 \%(\mathrm{~N}=518)$ patients.

\section{Statistical analysis}

Overall survival (OS) and freedom from failure (FF) were the primary endpoints of the study. Survival outcomes were measured from the completion of treatment. Failure was defined as cancer recurrence anywhere within the body, both locally within in the pelvis and distantly (sites outside of the radiotherapy field). BMI groups were determined by using existing clinical categories of obesity as defined by the World Health Organization. Outcome-oriented methods for determining cutpoints proposed by Contal \& O'Quigley were then used to determine groups within our patient population. [13] For analysis of all patients $(\mathrm{n}=591)$, patients were stratified into 3 BMI groups: $\mathrm{A} \leq$ 18.5; $\mathrm{B} 18.6$ - 34.9; and $\mathrm{C} \geq 35$. For the subset of patients undergoing mutational and $\mathrm{IHC}$ analysis, patients were stratified into only 2 groups (BMI less than or equal vs greater than 30) because of the smaller total number of patients in this subset. There were insufficient numbers of translational correlates in our institutional datasets from patients with $\mathrm{BMI}>35$ to provide meaningful statistical analysis. SAS v9.4 and R v3.0.3 were used for the analyses. $\mathrm{P}<0.05$ was set as the threshold for significance for all study outcomes. The Kaplan-Meier (product-limit) method and the Log-Rank test were used to derive time-to-event estimates and test for significance. Fisher's Exact test was utilized to compare differences between categorical data. ANOVA and Independent $t$-tests were utilized to compare continuous covariates and z-test for proportions was used to test differences in proportions. Multivariate proportional hazards modeling was performed as previously described [14]. The final model that was constructed consisted of variables clinical stage, lymph node status and cervical tumor SUV from the pretreatment FDG-PET and BMI > 35 .

\section{Immunohistochemistry analysis}

A tissue microarray (TMA) was constructed from pre-treatment formalin-fixed paraffin embedded (FFPE) tumor specimens. Punches were taken from marked areas for tumor content and used to construct a tissue microarray on MTA-1 Manual Tissue Arrayer (Beecher Instruments, Inc., Sun Prairie, WI). Slides were prepared from $0.6 \mu \mathrm{m}$ sections, deparaffinized and rehydrated per manufacturer's protocol. After antigen retrieval, slides were stained with anti-phosphoAKT antibody (Rabbit anti-human AKT-1 (phospho-S473) polyclonal, Spring Bioscience) per manufacturer's protocol using the Ventana BenchMark ULTRA autostainer (Ventana Medical Systems Inc., Tuscon, AZ) using cell conditioning 
step CC1. Slides were scanned and digitized on ScanScope XT (Leica Biosystems, Buffalo Grove, IL), and analyzed using the web-based Aperio eSlide Manager platform (Leica Biosystems, Buffalo Grove, IL). Staining was reviewed in a blinded fashion by two reviewers, and given a consensus score for intensity of pAKT staining as absent, weak, intermediate or strong intensity.

\section{$P I K 3 C A$ and $P T E N$ sequencing}

Tumor biopsies were sectioned and subjected to review by a pathologist. Only tumor specimens with $\geq$ $60 \%$ neoplastic cellularity and $<20 \%$ necrosis were used for further analysis. DNA was extracted from frozen tumor tissue using QIAamp DNA kit (Qiagen, USA) according to the manufacturer's instructions. Illumina libraries were constructed with dual-index barcode sequences and enrichment was performed for target regions as previously described [15]. Hybridization capture sequence data from tumor and matched normal DNA was used to catalogue somatic mutations in PIK3CA and PTEN, including loss of heterozygosity ( $\mathrm{LOH})$. More specifically, sequence data were aligned to GRCh37-lite_WUGSC_variant_2 (http://genome.wustl.edu/pub/reference/GRCh37-lite WUGSC_variant_2/) using bwa-mem (Processing Profile c3e6b636310547caaa9776e9aca5e4c5: bwamem-stream 0.7.10 [-t 8]). Bams were merged using Picard 1.113 then deduplicated using Picard 1.113 api v6. Putative somatic point mutations were detected using tumor and normal Samtools r982 [16], Varscan 2.3.6 [17], Strelka 1.0.11, [18] and Somatic Sniper [19]. Putative somatic indels were identified using GATK (gatk-somatic-indel 5336) [20], Pindel 0.5 [20] and Varscan 2.3.6. Variants were merged and filtered as previously described. In addition, putative variants were filtered to remove known germline dbSNPs (dbSNP 137), artifacts detected in a panel of normal samples [21] variants with less than 8 referenceallele-supporting reads in the Normal DNA, and less than two supporting reads or a less than of $10 \%$ variant allele fraction in the tumor. PTEN LOH predictions were made using VarScan 2.3.6 based on read coverage distribution differences between tumor and matched normal using methods previously published [17]. Mutation locations were mapped to a protein framework using the lolliplot function in GenVisR package (http://bioconductor.org/ package/GenVisR) implemented in R3.3.0.

\section{Reverse phase protein array data analysis}

Reverse Phase Protein Array (RPPA) Data was generated by the Cancer Genome Atlas (TCGA) Research Network [22]. Normalized level 4 RPPA data from cervical cancer were obtained from the Cancer Proteome Atlas [23]. Clinical data including weight, height, and survival was obtained from cbioPortal $[24,25]$. Log2 normalized protein levels were compared using a two tailed $t$-test with multiple comparison correction (Benjamini and Hochber alogrithm) using Matlab (MATLAB and Bioinformatics Toolkit 2015b, The MathWorks, Inc., Natick, Massachusetts, United States).

\section{Abbreviations}

$$
\begin{aligned}
& \text { BMI - Body Mass Index } \\
& \text { FDG }-{ }^{18} \mathrm{~F} \text { - fluoro-deoxy-glucose } \\
& \text { FF - freedom form failure } \\
& \text { FIGO - International Federation of Gynecology and } \\
& \text { Obstetrics } \\
& \text { HPV - human papilloma virus } \\
& \text { IHC - immunohistochemistry } \\
& \text { LN - pretreatment lymph node status } \\
& \text { MUT - mutant } \\
& \text { OS - overall survival } \\
& \text { pAKT - phosphorylated AKT } \\
& \text { PET - positron emission tomography } \\
& \text { RPPA - reverse phase protein array } \\
& \text { RT - radiation } \\
& \text { SUV - standardized uptake value } \\
& \text { TCGA - the Cancer Genome Atlas } \\
& \text { TMA - tissue microarray } \\
& \text { WT - wild type. }
\end{aligned}
$$

\section{Author contributions}

PG collects and maintains the institutional cervical cancer clinical outcomes database. PG, AC, ALT, TD and JKS collected and performed clinical data analysis. SM and JDP performed and analyzed immunohistochemistry. FR, MM, CM, RR, RF and JKS performed and analyzed DNA sequencing. AE performed TCGA data analysis. JKS wrote the manuscript. PG and SM provided critical feedback. All authors reviewed the manuscript.

\section{ACKNOWLEDGMENTS}

We thank Richard K. Wilson, Ph.D. and the McDonnell Genome Institute for DNA sequencing. We thank Drs. Li Ding, Amila Weerasinghe and Rehan Akbani for assistance with reverse phase protein array data analysis. We thank the Alvin J. Siteman Cancer Center at Washington University School of Medicine and Barnes-Jewish Hospital in St. Louis, MO, for the use of the Tissue Procurement Core, which provided tissue banking services.

\section{CONFLICTS OF INTEREST}

The authors report no conflicts of interest.

\section{FUNDING}

This research was supported by NIH R01 CA181745-01 (JKS). The Siteman Cancer Center is 
supported in part by an NCI Cancer Center Support Grant \#P30 CA91842. AE was supported by \#RMS1408 from the Radiological Society of North America (RSNA). FR was supported by the Siteman Cancer Center Cancer Biology Pathway Fellowship and Molecular Oncology Training Grant T32 CA113275.

\section{REFERENCES}

1. Calle EE, Rodriguez C, Walker-Thurmond K, Thun MJ. Overweight, obesity, and mortality from cancer in a prospectively studied cohort of U.S. adults. N Engl J Med. 2003; 348:1625-38. https://doi.org/10.1056/ NEJMoa021423.

2. Reeves GK, Pirie K, Beral V, Green J, Spencer E, Bull D; Million Women Study Collaboration. Cancer incidence and mortality in relation to body mass index in the million women study: cohort study. BMJ. 2007; 335:1134. https:// doi.org/10.1136/bmj.39367.495995.AE.

3. Xu X, Zhou L, Miao R, Chen W, Zhou Y, Pang Q, Qu K, Liu C. Association of cancer mortality with postdiagnosis overweight and obesity using body mass index. Oncotarget. 2016; 7:5023-9. https://doi.org/10.18632/oncotarget.6517.

4. Kidd EA, Siegel BA, Dehdashti F, Grigsby PW. The standardized uptake value for F-18 fluorodeoxyglucose is a sensitive predictive biomarker for cervical cancer treatment response and survival. Cancer. 2007; 110:1738-44. https:// doi.org/10.1002/cncr.22974.

5. Schwarz JK, Payton JE, Rashmi R, Xiang T, Jia Y, Huettner P, Rogers BE, Yang Q, Watson M, Rader JS, Grigsby PW. Pathway-specific analysis of gene expression data identifies the PI3K/Akt pathway as a novel therapeutic target in cervical cancer. Clin Cancer Res. 2012; 18:1464-71. https:// doi.org/10.1158/1078-0432.CCR-11-2485.

6. Rashmi R, DeSelm C, Helms C, Bowcock A, Rogers BE, Rader JL, Grigsby PW, Schwarz JK. AKT inhibitors promote cell death in cervical cancer through disruption of mTOR signaling and glucose uptake. PLoS One. 2014; 9:e92948. https://doi.org/10.1371/journal. pone. 0092948 .

7. Isakoff SJ, Engelman JA, Irie HY, Luo J, Brachmann SM, Pearline RV, Cantley LC, Brugge JS. Breast cancerassociated PIK3CA mutations are oncogenic in mammary epithelial cells. Cancer Res. 2005; 65:10992-1000. https:// doi.org/10.1158/0008-5472.CAN-05-2612.

8. Bader AG, Kang S, Vogt PK. Cancer-specific mutations in PIK3CA are oncogenic in vivo. Proc Natl Acad Sci U S A. 2006; 103:1475-9. https://doi.org/10.1073/ pnas.0510857103.

9. Zhao JJ, Liu Z, Wang L, Shin E, Loda MF, Roberts TM. The oncogenic properties of mutant p110alpha and p110beta phosphatidylinositol 3-kinases in human mammary epithelial cells. Proc Natl Acad Sci U S A. 2005; 102:18443-8. https://doi.org/10.1073/ pnas. 0508988102 .
10. Gymnopoulos M, Elsliger MA, Vogt PK. Rare cancerspecific mutations in PIK3CA show gain of function. Proc Natl Acad Sci U S A. 2007; 104:5569-74. https://doi. org/10.1073/pnas.0701005104.

11. Ocana A, Vera-Badillo F, Al-Mubarak M, Templeton AJ, Corrales-Sanchez V, Diez-Gonzalez L, Cuenca-Lopez MD, Seruga B, Pandiella A, Amir E. Activation of the $\mathrm{PI} 3 \mathrm{~K} / \mathrm{mTOR} / \mathrm{AKT}$ pathway and survival in solid tumors: systematic review and meta-analysis. PLoS One. 2014; 9:e95219. https://doi.org/10.1371/journal.pone.0095219.

12. Macdonald DM, Lin LL, Biehl K, Mutic S, Nantz R, Grigsby PW. Combined intensity-modulated radiation therapy and brachytherapy in the treatment of cervical cancer. Int J Radiat Oncol Biol Phys. 2008; 71:618-24. https://doi.org/10.1016/j.ijrobp.2008.02.014.

13. Contal C, O'Quigley J. An application of changepoint methods in studying the effect of age on survival in breast cancer. Comput Stat Data Anal. 1999; 30:253-70. https:// doi.org/10.1016/S0167-9473(98)00096-6.

14. Schwarz JK, Siegel BA, Dehdashti F, Grigsby PW. Association of posttherapy positron emission tomography with tumor response and survival in cervical carcinoma. JAMA. 2007; 298:2289-95. https://doi.org/10.1001/ jama.298.19.2289.

15. Griffith M, Miller CA, Griffith OL, Krysiak K, Skidmore ZL, Ramu A, Walker JR, Dang HX, Trani L, Larson DE, Demeter RT, Wendl MC, McMichael JF, et al. Optimizing cancer genome sequencing and analysis. Cell Syst. 2015; 1:210-23. https://doi.org/10.1016/j.cels.2015.08.015.

16. Li H, Handsaker B, Wysoker A, Fennell T, Ruan J, Homer N, Marth G, Abecasis G, Durbin R, Genome project data processing $\mathrm{S}$. The sequence alignment/map format and SAMtools. Bioinformatics. 2009; 25:2078-9. https://doi. org/10.1093/bioinformatics/btp352.

17. Koboldt DC, Zhang Q, Larson DE, Shen D, McLellan MD, Lin L, Miller CA, Mardis ER, Ding L, Wilson RK. Varscan 2: somatic mutation and copy number alteration discovery in cancer by exome sequencing. Genome Res. 2012; 22:568-76. https://doi.org/10.1101/gr.129684.111.

18. Saunders CT, Wong WS, Swamy S, Becq J, Murray LJ, Cheetham RK. Strelka: accurate somatic small-variant calling from sequenced tumor-normal sample pairs. Bioinformatics. 2012; 28:1811-7. https://doi.org/10.1093/ bioinformatics/bts271.

19. Larson DE, Harris CC, Chen K, Koboldt DC, Abbott TE, Dooling DJ, Ley TJ, Mardis ER, Wilson RK, Ding L. Somaticsniper: identification of somatic point mutations in whole genome sequencing data. Bioinformatics. 2012; 28:311-7. https://doi.org/10.1093/bioinformatics/btr665.

20. McKenna A, Hanna M, Banks E, Sivachenko A, Cibulskis K, Kernytsky A, Garimella K, Altshuler D, Gabriel S, Daly M, DePristo MA. The genome analysis toolkit: a mapreduce framework for analyzing next-generation DNA sequencing data. Genome Res. 2010; 20:1297-303. https:// doi.org/10.1101/gr.107524.110. 
21. Cancer Genome Atlas Network. Comprehensive molecular portraits of human breast tumours. Nature. 2012; 490:6170. https://doi.org/10.1038/nature11412.

22. Cancer Genome Atlas Research Network, Albert Einstein College of Medicine, Analytical Biological Services, Barretos Cancer Hospital, Baylor College of Medicine, Beckman Research Institute of City of Hope, Buck Institute for Research on Aging, Canada's Michael Smith Genome Sciences Centre, Harvard Medical School, Helen FGCC, Research Institute at Christiana Care Health Services, HudsonAlpha Institute for Biotechnology, Ilsbio LLC, et al. Integrated genomic and molecular characterization of cervical cancer. Nature. 2017; 543:378-84. https://doi. org/10.1038/nature21386.

23. Li J, Lu Y, Akbani R, Ju Z, Roebuck PL, Liu W, Yang JY, Broom BM, Verhaak RG, Kane DW, Wakefield C,
Weinstein JN, Mills GB, et al. TCPA: a resource for cancer functional proteomics data. Nat Methods. 2013; 10:1046-7. https://doi.org/10.1038/nmeth.2650.

24. Gao J, Aksoy BA, Dogrusoz U, Dresdner G, Gross B, Sumer SO, Sun Y, Jacobsen A, Sinha R, Larsson E, Cerami E, Sander C, Schultz N. Integrative analysis of complex cancer genomics and clinical profiles using the cBioPortal. Sci Signal. 2013; 6:pl1. https://doi.org/10.1126/ scisignal.2004088.

25. Cerami E, Gao J, Dogrusoz U, Gross BE, Sumer SO, Aksoy BA, Jacobsen A, Byrne CJ, Heuer ML, Larsson E, Antipin Y, Reva B, Goldberg AP, et al. The cBio cancer genomics portal: an open platform for exploring multidimensional cancer genomics data. Cancer Discov. 2012; 2:401-4. https://doi.org/10.1158/2159-8290.CD-12-0095. 